\title{
Utility of Neuropathy Screening for Wild-Type Transthyretin Amyloidosis Patients
}

\author{
Angela Russell, Christopher Hahn, Sameer Chhibber, Lawrence Korngut, \\ Nowell M. Fine
}

\begin{abstract}
Background: Wild-type transthyretin amyloidosis (wtATTR) is an important cause of heart failure (HF); however, the prevalence and clinical significance of neurologic complications remains uncertain. Methods: This analysis reports findings from a singlecentre experience of routine neuropathy screening at the time of wtATTR diagnosis by nerve conduction studies and neurologist assessment, compared with age-matched controls. Results: Forty-one wtATTR patients were included, 39 (95\%) males, mean age $78.4 \pm 7.7$ years, $22(54 \%)$ New York Heart Association (NYHA) class III-IV HF, along with 15 age-matched controls (mean age $77.1 \pm 4.2$ years, $80 \%$ male). Twenty-one (51\%) wtATTR patients were diagnosed with polyneuropathy, 15 (37\%) with spinal stenosis, $36(88 \%)$ with carpal tunnel syndrome (CTS) and 14 (34\%) with ulnar neuropathy. Comparison diagnoses among controls were $1(7 \%), 0$, $1(7 \%)$ and $3(20 \%)$, respectively. Among patients with NYHA class III-IV HF, 16 (73\%) had polyneuropathy compared with $5(26 \%)$ with class I-II ( $\mathrm{p}<0.01)$, odds ratio of 7.5 (95\% confidence interval 1.9-29.9). After neuropathy screening, 19 (46\%) patients were offered neurologic therapy and/or additional diagnostic evaluation. This included CTS release surgery (16, 39\%), neuropathic pain medication $(3,7 \%)$, nerve block $(1,2 \%)$, wrist splinting $(2,5 \%)$ and foot care $(1,2 \%)$. Spine imaging was performed for $3(7 \%)$ patients, and deltoid muscle and sural nerve biopsy for $1(2 \%)$ patient. Conclusions: Screening of wtATTR patients for neurologic complications resulted in a management change for nearly half. CTS, polyneuropathy and ulnar neuropathy were common. This approach warrants consideration as part of routine assessment for newly diagnosed wtATTR patients.
\end{abstract}

RÉSUMÉ : L'utilité du dépistage de neuropathies dans l'amylose à transthyrétine « sauvage ». Contexte : L'amylose à transthyrétine « sauvage » (ATS) est une cause importante d'insuffisance cardiaque (IC), mais on ne connaît pas très bien la prévalence ni la portée clinique des complications neurologiques. Méthode : Il s'agit d'une analyse unicentrique faisant état des résultats du dépistage systématique de neuropathies effectué chez des patients au moment de la pose du diagnostic d'ATS, et fondé sur des examens de la conduction nerveuse et des évaluations neurologiques faites par des spécialistes, par rapport aux résultats obtenus chez des témoins appariés selon l'âge. Résultats : Ont été retenus dans l'étude 41 patients atteints d'ATS : hommes : 39 (95\%); âge moyen : 78,4 \pm 7,7 ans; IC de classe III ou IV selon la New York Heart Association (NYHA) : 22 patients (54\%); ainsi que 15 témoins appariés selon l'âge : âge moyen : 77,1 $\pm 4,2$ ans; hommes : $80 \%$. Un diagnostic de polyneuropathie a été posé chez 21 patients atteints d'ATS (51\%); de sténose du canal rachidien, chez 15 patients (37\%); de syndrome du canal carpien (SCC), chez 36 patients (88\%) et de neuropathie cubitale, chez 14 patients (34\%); par comparaison, ces diagnostics ont été posés respectivement chez 1 témoin (7\%), 0 témoin, 1 témoin (7\%) et 3 témoins (20\%). Parmi les patients souffrant d'IC de classe III ou IV selon la NYHA, 16 (73\%) présentaient des signes de polyneuropathie contre 5 patients (26 \%) souffrant d'IC de classe I ou II ( $p<0,01)$, soit un risque relatif approché de 7,5 (intervalle de confiance à 95 \%: 1,9-29,9). À la suite du dépistage de neuropathies, 19 patients (46\%) se sont vu offrir des formes additionnelles de traitement neurologique ou d'évaluation diagnostique, notamment une opération de décompression du nerf médian dans le SCC (16;39\%); des analgésiques contre la douleur neuropathique (3; $7 \%)$; une analgésie par blocage nerveux $(1 ; 2 \%)$; le port d'une orthèse de poignet $(2 ; 5 \%)$ ou des soins podologiques $(1 ; 2 \%)$. Des examens par imagerie de la colonne vertébrale ont été effectués chez 3 patients $(7 \%)$ et une biopsie du muscle deltoïde et du nerf saphène externe, chez 1 patient $(2 \%)$. Conclusion : Le dépistage de complications neurologiques chez les patients atteints d'ATS s'est traduit par une modification de la prise en charge de la maladie chez près de la moitié des sujets. Au nombre des complications fréquentes figuraient le SCC, la polyneuropathie et la neuropathie cubitale. Aussi serait-il justifié d'intégrer le dépistage neurologique dans l'évaluation courante des patients chez qui un diagnostic d'ATS vient d'être posé.

Keywords: Wild-type transthyretin amyloidosis, Neuropathy, Diagnosis, Management doi:10.1017/cjn.2020.271

Can J Neurol Sci. 2021; 48: 607-615

\section{INTRODUCTION}

Wild-type transthyretin amyloidosis (wtATTR) is caused by misfolded native (non-mutated) transthyretin (TTR) proteins that deposit in the interstitium of organ tissues. ${ }^{1,2}$ Unlike hereditary ATTR (hATTR, caused by a TTR gene mutation) which can occur in younger patients and may have a predominant neuropathic

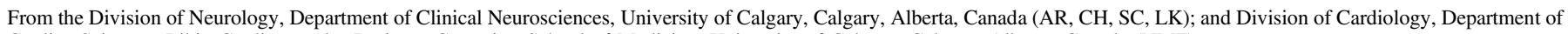
Cardiac Sciences, Libin Cardiovascular Institute, Cumming School of Medicine, University of Calgary, Calgary, Alberta, Canada (NMF)

Received November 16, 2020. Final Revisions Submitted December 9, 2020. Date of Acceptance December 10, 2020.

Correspondence to: Nowell M. Fine, South Health Campus, 4448 Front Street Southeast, Calgary, AB, T3M 1M4, Canada. Email: nmfine@ucalgary.ca 
phenotype, wtATTR is most often a disease of older men that primarily causes a slowly progressive infiltrative cardiomyopathy. ${ }^{1,2}$

The burden of peripheral neuropathy in wtATTR is regarded to be less than that of other amyloidosis subtypes, ${ }^{2}$ and the natural history of wtATTR associated with peripheral neuropathy is poorly understood. Carpal tunnel syndrome (CTS) is one of the most common extra-cardiac manifestations of wtATTR and can predate cardiac symptoms by many years. ${ }^{3}$ The prevalence of symptomatic CTS is thought to be higher in wtATTR patients compared to light chain (AL) amyloidosis. ${ }^{4}$ Amyloid deposition in the ligamentum flavum has also been observed in wtATTR, resulting in symptomatic spinal stenosis. ${ }^{5}$ Few studies have commented on the prevalence of symptomatic polyneuropathy in wtATTR, ${ }^{4,6,7}$ and no studies have addressed asymptomatic neuropathy. This may in part be due to the increased age and multiple comorbidities common to this population. Similarly, the prevalence of other entrapment neuropathies, such as ulnar neuropathy at the elbow, has never been reported.

At our centre, all newly diagnosed wtATTR patients undergo multidisciplinary evaluation for extra-cardiac disease manifestations as part of a standardised clinical protocol, inclusive of routine neurologic assessment. The purpose of our study was to evaluate the yield of this routine neurologic screening approach in wtATTR patients, including the prevalence of peripheral neuropathy and its potential role in interventions.

\section{MethodS}

\section{Study Population}

Consecutive patients with a diagnosis of wtATTR followed at the University of Calgary Cardiac Amyloidosis Clinic (Calgary, Alberta, Canada) between February 2014 and October 2019 were included in this retrospective cohort study. All patients with a diagnosis of wtATTR followed in this clinic were routinely referred for neuropathy screening. A diagnosis of wtATTR was confirmed using the following criteria: (1) exclusion of AL amyloidosis by the absence of serum and urine monoclonal protein, (2) evidence of cardiac amyloidosis by either right ventricular endomyocardial biopsy or positive technetium-99 m-pyrophosphate nuclear scintigraphy, defined as grade 2-3 myocardial uptake or heart-to-contralateral lung ratio $>1.5$, as previously described ${ }^{8}$ and 3 ) absence of an ATTR gene mutation using genetic testing or proteomic analysis by mass spectrometry performed on biopsy tissue sample. ${ }^{9}$ Neuropathy screening included nerve conduction studies (NCS) with electromyography (EMG) when indicated and comprehensive clinical assessment by a neurologist during the electrophysiologic testing visit inclusive of history and physical examination (Figure 1).

Fifteen age-matched controls without ATTR referred for NCS and EMG evaluation for the indication of possible myasthenia gravis without generalised weakness were selected as a comparator group, as the incidence of polyneuropathy, CTS and ulnar neuropathy specific to this age group is not well described. These patients were selected because as a neuromuscular junction disease, NCS results are not significantly affected by myasthenia gravis. Only two of the controls were diagnosed with mild myasthenia gravis, while the remainder were not. Additionally, for the purpose of comparison with wtATTR patients, only control patients without the following comorbid conditions commonly associated with peripheral neuropathy were included diabetes mellitus, thyroid dysfunction, monoclonal gammopathy of undetermined significance, haematologic malignancy, vitamin $\mathrm{B}_{12}, \mathrm{~B}_{6}$, and $\mathrm{B}_{1}$ deficiencies, folic acid deficiency, alcohol abuse, renal failure, history of chemotherapy and inherited neuropathy. All controls had both upper and lower extremity electrodiagnostic studies performed, similar to wtATTR patients. This study was approved by the University of Calgary Research Ethics Board, and the requirement for informed patient consent was waived.

\section{Electrodiagnostics}

Comprehensive electrodiagnostic evaluation was performed in a dedicated and accredited laboratory by an experienced neurologist who also performed a complete clinical assessment including history and physical examination during the visit (Figure 1). Standard of practice for our laboratory is to record unilateral median, ulnar, radial, superficial fibular and sural sensory nerve action potentials (SNAPs), and median (abductor pollicis brevis), ulnar (abductor digiti minimi), tibial (abductor hallicus) and fibular (extensor digitorum brevis) compound muscle action potentials (CMAPs). Peak-to-baseline measurements were used. Recording sites, inter-electrode distance and temperature were all standardised as per laboratory protocol. Bilateral studies are done only if asymmetry is noted on physical examination or history at the discretion of the neurologist. F-waves were also done at the discretion of the performing neurologist. Patients with suspected CTS or ulnar neuropathy have bilateral ulnar and median SNAPs/ CMAPs done by protocol including median versus ulnar digit 4 comparison studies. Additional median to other nerve comparison studies were done when needed as per the 2011 American Association of Neuromuscular \& Electrodiagnostic Medicine (AANEM) monograph on electrodiagnostic evaluation of CTS. ${ }^{10}$ The evaluation of ulnar neuropathy was based on the 1999 AANEM practice parameter for electrodiagnostic studies in ulnar neuropathy at the elbow. ${ }^{11}$ Testing was performed using either a Cadwell Sierra Wave machine running version 3.1.32 of Cadlink or Natus Ultrapro S100 machine running version 22.3.0.21 of Synergy.

\section{Data Collection}

Medical records of ATTR patients were reviewed for a prior diagnosis of peripheral neuropathy. Comorbidities commonly associated with peripheral neuropathy were collected, which included diabetes mellitus, thyroid dysfunction, haematologic malignancy, monoclonal gammopathy of undetermined significance, vitamin $\mathrm{B}_{12}, \mathrm{~B}_{6}$ and $\mathrm{B}_{1}$ deficiencies, folic acid deficiency, alcohol abuse, renal failure, history of chemotherapy and inherited neuropathy. Symptoms of mononeuropathy (CTS and ulnar) and polyneuropathy (numbness, tingling, neuropathic pain and weakness) were also collected, along with the following physical examination findings suggestive of polyneuropathy: stocking/glove sensory loss, impaired or absent vibration sensation, reduced or absent ankle reflexes, distal muscle atrophy (bilateral), and extensor hallucis longus, ankle dorsiflexion and ankle plantar flexion weakness (bilateral). Electrodiagnostic data collected included radial SNAP amplitude, tibial CMAP amplitude, sural SNAP amplitude, fibular CMAP amplitude and superficial fibular SNAP amplitude. In cases where bilateral studies were done, the side with the highest SNAP or CMAP value was recorded. Neurologist diagnoses and CTS electrodiagnostic severity as per the 2011 AANEM monograph were recorded. ${ }^{10}$ Baseline cardiovascular data collected included New York Heart Association (NYHA) functional class in addition to medication, 


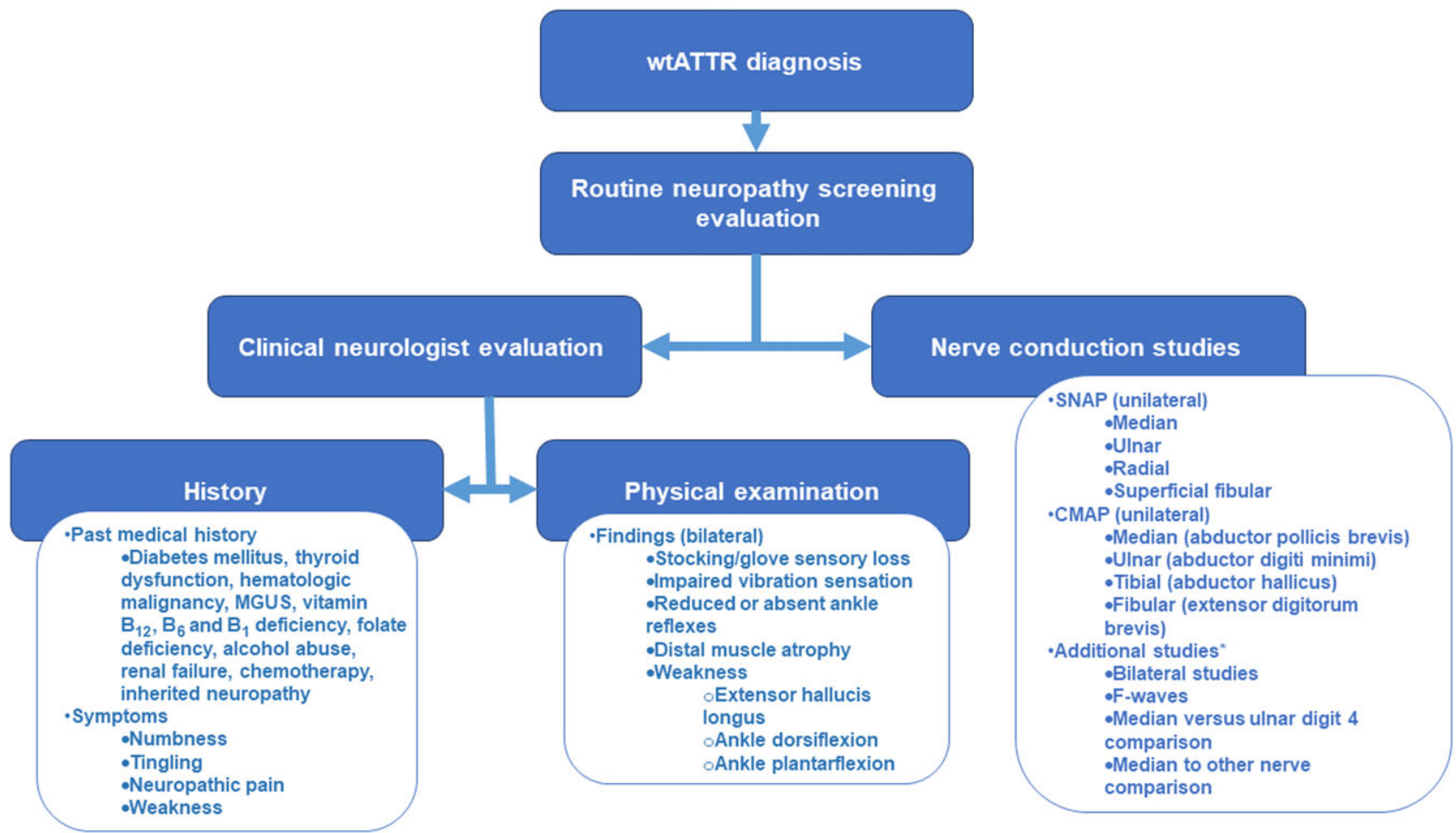

Figure 1: Flowchart describing the diagnostic approach used for routine neuropathy screening of wtATTR patients. *Additional studies were done at the discretion of the electromyographer based on the following criteria: bilateral studies were done only if asymmetry was noted on history or physical examination. Patients with symptoms, physical examination findings or NCS findings consistent with CTS or ulnar neuropathy underwent bilateral studies including median versus ulnar digit 4 comparison studies. F-waves and median to other nerve comparison studies were done at the discretion of the performing physician. CTS, carpal tunnel syndrome; wtATTR, wild-type transthyretin amyloidosis; NCS, nerve conduction studies; MGUS, monoclonal gammopathy of undetermined significance.

biochemical (troponin-T, N-terminal pro-B-type natriuretic peptide [NTproBNP]) and echocardiographic data.

For the purposes of this analysis, electrodiagnostic evidence of polyneuropathy was defined as an abnormality of any nerve conduction SNAP or CMAP amplitude in two separate nerves, one of which must be the sural nerve. ${ }^{12}$ Normal values were obtained using published reference standards. ${ }^{13-15}$ Amplitudes below $1 \mu \mathrm{V}$ were confirmed by visual waveform analysis. The subtype of polyneuropathy was determined by the assessing neurologist, based on electrodiagnostic abnormalities, patient symptoms and physical examination findings. In this study, a diagnosis of polyneuropathy was made based on the electrodiagnostic criteria outlined above, along with either symptoms or physical examination findings of polyneuropathy, also outlined above. A minority of patients received a diagnosis of polyneuropathy from the assessing neurologist but did not meet electrodiagnostic criteria. These patients had either one abnormal NCS amplitude and physical examination findings or nondiagnostic NCS amplitudes, but both suggestive symptoms and at least two physical examination findings. Distinguishing polyneuropathy without compatible NCS findings from other causes was made on clinical grounds, based on complete history and physical examination. Pure small fibre polyneuropathy was defined as impaired pain and temperature sensation in a stocking and glove or nonlength-dependant distribution, with preserved motor function, deep tendon reflexes, vibration sensation and proprioception. The modified polyneuropathy disability (PND) score was used to assess the severity of polyneuropathy in wtATTR patients. ${ }^{16,17}$ A PND score of I or II suggests mild polyneuropathy, III or IV suggests moderate polyneuropathy and V suggests severe polyneuropathy. Diagnosis of CTS and ulnar neuropathy were based on neurologist interpretation of patient history, physical examination and electrodiagnostic data. The electrodiagnostic criteria for CTS and ulnar neuropathy at the elbow were derived from the AANEM practice parameters. ${ }^{10,11}$ Data were also collected on the need for follow-up neurologic evaluation, additional diagnostic investigations performed and type of therapeutic intervention offered resulting from screening neurologic assessment.

\section{Statistical Analysis}

Categorical variables are presented as absolute values and percentages, while continuous variables are expressed as mean \pm standard deviation or median (interquartile range). Categorical variables were compared using the Fisher's exact test, while comparisons for continuous data were performed using the two-sample independent t-test or Wilcoxon rank-sum test, where appropriate. Odds ratios ( $95 \%$ confidence interval) for factors associated with the diagnosis of polyneuropathy were calculated. Correction for multiple comparisons was performed using the Bonferroni method. All statistical analyses were performed using commercially available software (Stata, version 14.2, StataCorp., College Station, Texas). A two-sided p-value of less than 0.05 was considered statistically significant. 


\section{Table 1: Baseline patient characteristics}

\begin{tabular}{|c|c|c|c|c|}
\hline & \multicolumn{3}{|c|}{ wtATTR } & \multirow[b]{2}{*}{ Controls } \\
\hline & All & $\begin{array}{c}\text { Excluding comorbidities } \\
\text { associated with polyneuropathy }\end{array}$ & $\begin{array}{l}\text { With comorbidities associated } \\
\text { with polyneuropathy }\end{array}$ & \\
\hline Sample size & 41 & 27 & 14 & 15 \\
\hline \multicolumn{5}{|l|}{ Clinical characteristics } \\
\hline Age (years) & $78.4 \pm 7.7$ & $78.1 \pm 6.1$ & $78.8 \pm 8.4$ & $77.1 \pm 4.2$ \\
\hline Male & $39(95 \%)$ & $27(100 \%)$ & $12(86 \%)$ & $12(80 \%)$ \\
\hline Heart rate (beats/minute) & $72 \pm 15$ & $69 \pm 17$ & $74 \pm 12$ & \\
\hline Systolic BP (mmHg) & $106 \pm 34$ & $113 \pm 29$ & $101 \pm 39$ & \\
\hline Diastolic BP (mmHg) & $68 \pm 21$ & $61 \pm 26$ & $74 \pm 18$ & \\
\hline NYHA functional class III-IV & $22(54 \%)$ & $14(52 \%)$ & $8(57 \%)$ & \\
\hline \multicolumn{5}{|l|}{ Comorbidities } \\
\hline Diabetes & $4(10 \%)$ & & $4(29 \%)$ & \\
\hline Thyroid dysfunction & $4(10 \%)$ & & $4(29 \%)$ & \\
\hline MGUS/haeme malignancy & $2(5 \%)$ & & $2(14 \%)$ & \\
\hline $\mathrm{B}_{12}, \mathrm{~B}_{6}$ or $\mathrm{B}_{1}$ deficiency & 0 & & 0 & \\
\hline Folic acid deficiency & 0 & & 0 & \\
\hline Alcohol misuse & $1(2 \%)$ & & $1(7 \%)$ & \\
\hline Renal failure & $4(10 \%)$ & & $4(29 \%)$ & \\
\hline \multicolumn{5}{|l|}{ Medications } \\
\hline Diuretics & $35(85 \%)$ & $21(78 \%)$ & $14(100 \%)$ & \\
\hline Anticoagulants & $18(44 \%)$ & $1037 \%)$ & $8(57 \%)$ & \\
\hline \multicolumn{5}{|l|}{ Cardiac parameters } \\
\hline LV ejection fraction (\%) & $51 \pm 9$ & $54 \pm 11$ & $47 \pm 8$ & \\
\hline Troponin-T (ng/L) & $46 \pm 21$ & $38 \pm 26$ & $53 \pm 19$ & \\
\hline NTproBNP (ng/L) & $1928 \pm 853$ & $1732 \pm 937$ & $2409 \pm 715$ & \\
\hline
\end{tabular}

$\mathrm{BP}$, blood pressure; LV, left ventricle; MGUS, monoclonal gammopathy of undetermined significance; NTproBNP, N-terminal pro-B-type natriuretic peptide; NYHA, New York Heart Association; wtATTR, wild-type transthyretin amyloidosis.

\section{Results}

\section{Patient Population and Characteristics}

A total of 51 wtATTR patients were eligible for inclusion. Eight patients declined neuropathy screening and 2 had incomplete examinations, for a final study population of 41. Demographic, clinical, medication and cardiac characteristics are presented in Table 1, along with demographic characteristics of the 15 age-matched control patients. The majority of wtATTR patients (54\%) were NYHA functional class III-IV.

\section{Neurologic Evaluation}

Neurologic symptoms and physical examination findings for wtATTR patients and controls diagnosed with neuropathy are presented in Table 2. NCS amplitudes for wtATTR patients and controls are presented in Table 3, along with normative values. Diagnoses resulting from neurologic evaluation of wtATTR patients and controls are presented in Table 4. Figure 2 presents the frequency of prior (pre-existing) and new (diagnosed at the time of screening) neurologic diagnoses among wtATTR patients.

\section{Polyneuropathy}

There were six (15\% of all) wtATTR patients with a prior diagnosis of polyneuropathy that preceded wtATTR diagnosis. Fifteen (37\%) wtATTR patients received a new diagnosis of polyneuropathy as a result of routine screening. Eight of the 15 met electrodiagnostic criteria for polyneuropathy, while 4 had 1 abnormal NCS amplitude and suggestive physical examination findings and 3 patients received a diagnosis by the assessing neurologist using symptom history and at least 2 physical examination findings without meeting electrodiagnostic criteria. The majority of wtATTR patients with a diagnosis of polyneuropathy had a pure sensory polyneuropathy $(12,57 \%$ of those with polyneuropathy), while the remainder had sensorimotor polyneuropathy $(9,43 \%)$. No patients had a pure small fibre polyneuropathy. The most common symptom in patients diagnosed with polyneuropathy was numbness $(9,43 \%)$, while the most common physical examination findings were stocking/glove sensory loss $(17,81 \%)$ and reduced or absent ankle reflexes $(17,81 \%)$. Nine $(43 \%)$ patients diagnosed with polyneuropathy were asymptomatic. Thirteen (62\%) of the 21 patients diagnosed with polyneuropathy did not have a 
Table 2: Neurologic symptoms and physical examination findings for wtATTR patients and controls diagnosed with neuropathy

\begin{tabular}{|c|c|c|c|c|}
\hline & \multicolumn{3}{|c|}{ wtATTR } & \multirow[b]{2}{*}{ Controls } \\
\hline & All & $\begin{array}{l}\text { Excluding comorbidities associated } \\
\text { with polyneuropathy }\end{array}$ & $\begin{array}{c}\text { With comorbidities associated with } \\
\text { polyneuropathy }\end{array}$ & \\
\hline Sample size & 41 & 27 & 14 & 15 \\
\hline Polyneuropathy & $21(51 \%)$ & $13(48 \%)$ & $8(57 \%)$ & $1(7 \%)$ \\
\hline \multicolumn{5}{|l|}{ Symptoms } \\
\hline Numbness & $9(22 \%)$ & $5(19 \%)$ & $4(29 \%)$ & 0 \\
\hline Tingling & $6(15 \%)$ & $5(19 \%)$ & $1(7 \%)$ & 0 \\
\hline Neuropathic pain & $3(7 \%)$ & $1(4 \%)$ & $2(14 \%)$ & 0 \\
\hline None & $9(22 \%)$ & $6(22 \%)$ & $3(21 \%)$ & $1(7 \%)$ \\
\hline \multicolumn{5}{|l|}{ Physical exam findings } \\
\hline Stocking/glove sensory loss & $17(41 \%)$ & $9(33 \%)$ & $8(57 \%)$ & 0 \\
\hline Vibration impaired or absent & $15(37 \%)$ & $9(33 \%)$ & $6(43 \%)$ & $1(7 \%)$ \\
\hline Reduced or absent ankle reflexes & $17(41 \%)$ & $12(44 \%)$ & $5(36 \%)$ & $1(7 \%)$ \\
\hline Distal muscle atrophy (bilateral) & $3(7 \%)$ & $2(7 \%)$ & $1(7 \%)$ & 0 \\
\hline EHL/FDF weakness (bilateral) & $1(2 \%)$ & 0 & $1(7 \%)$ & 0 \\
\hline FPF weakness (bilateral) & 0 & 0 & 0 & 0 \\
\hline None & $1(2 \%)$ & $1(4 \%)$ & 0 & 0 \\
\hline \multicolumn{5}{|l|}{ Mononeuropathy } \\
\hline CTS & $36(88 \%)$ & $24(89 \%)$ & $12(86 \%)$ & $1(7 \%)$ \\
\hline Symptomatic & $28(68 \%)$ & $22(81 \%)$ & $6(43 \%)$ & 0 \\
\hline Asymptomatic & $8(20 \%)$ & $2(7 \%)$ & $6(43 \%)$ & $1(7 \%)$ \\
\hline Ulnar neuropathy & $14(34 \%)$ & $11(41 \%)$ & $3(21 \%)$ & $3(20 \%)$ \\
\hline Symptomatic & $3(7 \%)$ & $2(7 \%)$ & $1(7 \%)$ & 0 \\
\hline Asymptomatic & $11(27 \%)$ & $9(33 \%)$ & $2(14 \%)$ & $3(20 \%)$ \\
\hline
\end{tabular}

CTS, carpal tunnel syndrome; EHL, extensor hallucis longus; FDF, foot dorsiflexion; FPF, foot plantarflexion; wtATTR, wild-type transthyretin amyloidosis.

comorbidity commonly associated with polyneuropathy (Table 2). As assessed by PND score (median score I, range I-IV), 12 (57\%) patients had mild polyneuropathy, $4(19 \%)$ had moderate disease and none had severe polyneuropathy, while PND could not be calculated for 5 patients. Among 22 wtATTR patients with NYHA functional class III-IV heart failure (HF), 16 (73\%) had polyneuropathy. By comparison, of the 19 wtATTR patients with NYHA functional class I-II HF, 5 (26\%) had polyneuropathy ( $\mathrm{p}<0.01)$, for an odds ratio of 7.5 (95\% CI 1.9-29.9). Among the 16 wtATTR patients with polyneuropathy and available PND score, all 4 with moderate polyneuropathy also had NYHA functional class III-IV $\mathrm{HF}$, while among the 12 with mild polyneuropathy, 7 were NYHA class I-II and 5 were NYHA III-IV $(\mathrm{p}=0.09)$.

\section{Spinal Stenosis}

There were nine $(22 \%)$ patients with a prior history of spinal stenosis, including eight $(20 \%)$ with lumbar and one $(2 \%)$ with cervical stenosis and six $(15 \%)$ had prior spine surgery. Six $(15 \%)$ patients had a new diagnosis of spinal stenosis and three $(7 \%)$ had a new diagnosis of lumbar radiculopathy following neurologic screening. These diagnoses were made by either computed tomography scan or magnetic resonance imaging (MRI) studies. Asymptomatic patients did not undergo spine imaging.

\section{Carpal Tunnel Syndrome}

As anticipated, CTS was more common in wtATTR patients compared with controls (36, $88 \%$ vs. 1, 7\%, respectively, $\mathrm{p}<0.01$ ) (Table 4). The majority of wtATTR patients with CTS were symptomatic $(28,78 \%)$, compared to the single asymptomatic control $(\mathrm{p}=0.99)$. There were $18(44 \%$ of all $)$ wtATTR patients with a prior diagnosis of CTS, including $12(67 \%)$ with bilateral CTS. Of the 18 patients with a prior history of CTS, 13 (32\% of all wtATTR patients) had prior carpal tunnel release surgery. Most of these patients $(10,77 \%)$ had previous bilateral CTS release surgery. There were 21 (54\% of all) patients with a new diagnosis of CTS, including 3 (14\%) with mild, 10 (48\%) with moderate and $8(38 \%)$ with severe CTS. There were $14(34 \%$ of all) wtATTR patients with recurrent CTS, including one (7\%) with mild recurrent, eight $(57 \%)$ with moderate and two (14\%) with severe recurrent CTS; severity was not reported on three patients. Five (12\%) patients had recurrent bilateral CTS. Two patients reported symptoms of CTS but did not meet the criteria for diagnosis.

\section{Ulnar Neuropathy}

Ulnar neuropathy was also more common in wtATTR patients versus controls $(14,34 \%$ vs. $3,20 \%$, respectively, $\mathrm{p}=0.51)$ 
Table 3: NCS amplitudes for wtATTR patients and controls with age-specific normative values

\begin{tabular}{|c|c|c|c|c|c|c|c|c|}
\hline Nerve & Age range(years) & $\begin{array}{l}\text { All wtATTR } \\
\text { patients }\end{array}$ & $\begin{array}{c}\text { wtATTR } \\
\text { patients } \\
\text { excluding } \\
\text { comorbidities } \\
\text { associated with } \\
\text { polyneuropathy }\end{array}$ & $\begin{array}{c}\text { wtATTR } \\
\text { patients with } \\
\text { comorbidities } \\
\text { associated with } \\
\text { polyneuropathy }\end{array}$ & $\begin{array}{c}\text { wtATTR } \\
\text { patients with } \\
\text { diagnosed } \\
\text { polyneuropathy }\end{array}$ & $\begin{array}{c}\text { wtATTR } \\
\text { patients not } \\
\text { diagnosed with } \\
\text { polyneuropathy }\end{array}$ & Controls & $\begin{array}{l}\text { Normative } \\
\text { values }^{13-15}\end{array}$ \\
\hline Sample size & & 41 & 27 & 14 & 21 & 20 & 15 & \\
\hline \multicolumn{9}{|l|}{ Radial SNAP } \\
\hline & $\leq 79$ & $18.71 \pm 6.79$ & $19.5 \pm 6.56$ & $16.79 \pm 7.47$ & $16.43 \pm 7.52$ & $21.4 \pm 4.84$ & $32.25 \pm 15.01$ & $\geq 11 \mu \mathrm{V}$ \\
\hline & $80-89$ & $17.98 \pm 8.14$ & $18.14 \pm 8.05$ & $17.7 \pm 8.4$ & $14.98 \pm 9.74$ & $20.54 \pm 6.06$ & $22.13 \pm 10.08$ & $\geq 11 \mu \mathrm{V}$ \\
\hline & $\geq 90$ & $18.18 \pm 3.41$ & $17.65 \pm 1.34$ & $18.03 \pm 4.16$ & $19.7 \pm 4.24$ & $16.55 \pm 2.76$ & & $\geq 11 \mu \mathrm{V}$ \\
\hline \multicolumn{9}{|l|}{ Tibial motor } \\
\hline & $\leq 79$ & $5.89 \pm 4.29$ & $5.37 \pm 3.88$ & $7.14 \pm 5.28$ & $5.81 \pm 4.67$ & $5.98 \pm 4.02$ & $7.95 \pm 2.06$ & $\geq 1.1 \mathrm{mV}$ \\
\hline & $80-89$ & $4.35 \pm 2.86$ & $4.56 \pm 2.92$ & $4.03 \pm 2.62$ & $2.85 \pm 3.05$ & $5.64 \pm 2.09$ & $5.35 \pm 2.97$ & $\geq 0.2 \mathrm{mV}$ \\
\hline & $\geq 90$ & $5.83 \pm 4.47$ & $6.05 \pm 6.15$ & $4.3 \pm 3.99$ & $2 \pm 0.42$ & $9.65 \pm 1.06$ & & $\geq 0.2 \mathrm{mV}$ \\
\hline \multicolumn{9}{|l|}{ Sural SNAP } \\
\hline & $\leq 79$ & $3.65 \pm 3.36$ & $3.62 \pm 3.26$ & $3.73 \pm 3.86$ & $1.58 \pm 2.25$ & $6.11 \pm 2.56$ & $8.23 \pm 3.82$ & $\geq 4 \mu \mathrm{V}$ \\
\hline & $80-89$ & $4.37 \pm 5.18$ & $4.54 \pm 5.76$ & $7.48 \pm 7.83$ & $1.42 \pm 2.40$ & $6.9 \pm 5.71$ & $3.25 \pm 1.65$ & $\geq 0.5 \mu \mathrm{V}$ \\
\hline & $\geq 90$ & $1.4 \pm 1.78$ & $0.95 \pm 1.34$ & $1.23 \pm 2.14$ & 0 & $2.8 \pm 1.27$ & & $\geq 0.5 \mu \mathrm{V}$ \\
\hline \multicolumn{9}{|l|}{ Fibular motor } \\
\hline & $\leq 79$ & $2.68 \pm 1.8$ & $2.34 \pm 1.79$ & $3.51 \pm 1.64$ & $2.44 \pm 2.02$ & $2.96 \pm 1.54$ & $4.28 \pm 2.03$ & $\geq 1.1 \mathrm{mV}$ \\
\hline & $80-89$ & $2.09 \pm 1.92$ & $1.94 \pm 1.70$ & $2.35 \pm 2.46$ & $1.43 \pm 2.19$ & $2.66 \pm 1.60$ & $4.0 \pm 0.81$ & $0 \mathrm{mV}$ \\
\hline & $\geq 90$ & $1.75 \pm 0.94$ & $2.3 \pm 1.13$ & $1.3 \pm 0.35$ & $1.5 \pm 0$ & $2 \pm 1.56$ & & $0 \mathrm{mV}$ \\
\hline
\end{tabular}

NCS, nerve conduction study; SNAP, sensory nerve action potential; wtATTR, wild-type transthyretin amyloidosis.

(Table 4). The majority of wtATTR patients with ulnar neuropathy $(11,79 \%)$ had asymptomatic disease, represented a new diagnosis $(13,93 \%)$, and were unilateral $(9,64 \%)$. All controls with ulnar neuropathy were asymptomatic.

\section{Interventions and Implications for Management}

As a result of screening neurologic assessment, a total of 19 (46\%) of wtATTR patients were recommended additional diagnostic evaluation and/or subsequent therapy. Carpal tunnel release surgery was the most frequently recommended therapy $(16,39 \%$ of all wtATTR patients). Among these, five (12\%) patients underwent surgery, three $(7 \%)$ of whom received bilateral release surgery. There were three (7\%) patients who underwent additional investigations following screening. All had subsequent imaging examination and one (2\%) underwent nerve and muscle biopsy in addition to imaging. One patient had a cervical spine MRI that showed severe spinal stenosis with cord impingement. This patient was referred to a spine surgeon along with occupational and physical therapy; surgery was offered but declined by the patient. Another patient had an MRI of the cervical spine for possible cervical radiculopathy, which showed multilevel neural foraminal narrowing but no radiculopathy. The third patient declined MRI of the lumbar spine for probable lumbar radiculopathy and was referred for nerve and muscle biopsy for suspected amyloid neuromyopathy. Deltoid biopsy showed perivascular and perimysial amyloid deposition; sural nerve biopsy showed perivascular amyloid deposition and active chronic axonopathy with secondary active chronic demyelination, predominantly affecting unmyelinated fibres. Following screening, three $(7 \%)$ patients were prescribed medication(s) for neuropathic pain, which included gabapentin, duloxetine and capsaicin cream. One (2\%) patient received a nerve block for lumbar radiculopathy. Regarding other types of interventions, two (5\%) were prescribed wrist splinting for CTS, one (2\%) foot care for polyneuropathy (routine foot assessment and supportive footwear) and one (2\%) lifestyle modification for ulnar neuropathy (avoidance of direct pressure, over-extension or flexion of the elbow; elbow pads as needed). The number of patients who required ongoing neurologist clinical follow-up after initial routine screening evaluation was 14 (34\%).

\section{Discussion}

Our study shows that neuropathy is common in wtATTR patients and suggests that routine neurologic screening including NCS is warranted in this population. We observed that the prevalence of polyneuropathy in wtATTR patients was higher than anticipated $(>50 \%)$. Most were symptomatic and had a mild pure sensory polyneuropathy. The prevalence of CTS and ulnar neuropathy was also notable, with a significant proportion having no prior diagnosis. Most patients with CTS were symptomatic, while most with ulnar neuropathy were asymptomatic. The majority of patients with CTS had moderate unilateral disease, and recurrence after carpal tunnel release surgery was common (34\% of all). As a result of neuropathy screening, nearly half of all wtATTR patients were offered therapeutic intervention and/or further diagnostic evaluation; a significant proportion was 
Table 4: Diagnoses resulting from neuropathy screening in wtATTR patients and controls

\begin{tabular}{|c|c|c|c|c|c|}
\hline & \multicolumn{2}{|c|}{ wtATTR } & \multirow[b]{2}{*}{$\begin{array}{l}\text { With comorbidities } \\
\text { associated with } \\
\text { polyneuropathy } \\
\end{array}$} & \multirow[b]{2}{*}{ Controls } & \multirow[b]{2}{*}{ p-value } \\
\hline & All & $\begin{array}{l}\text { Excluding comorbidities } \\
\text { associated with } \\
\text { polyneuropathy }\end{array}$ & & & \\
\hline Sample size & 41 & 27 & 14 & 15 & \\
\hline \multicolumn{6}{|l|}{ Polyneuropathy } \\
\hline Any polyneuropathy & $21(51 \%)$ & $13(48 \%)$ & $8(57 \%)$ & $1(7 \%)$ & $<0.01$ \\
\hline Sensory & $12(29 \%)$ & $7(26 \%)$ & $5(36 \%)$ & $1(7 \%)$ & 0.22 \\
\hline Sensorimotor & $9(22 \%)$ & $6(22 \%)$ & $3(21 \%)$ & 0 & 0.07 \\
\hline \multicolumn{6}{|l|}{ CTS } \\
\hline Any CTS & $36(88 \%)$ & $24(89 \%)$ & $12(86 \%)$ & $1(7 \%)$ & $<0.01$ \\
\hline $\begin{array}{l}\text { Onset before wtATTR } \\
\text { diagnosis }\end{array}$ & $18(44 \%)$ & $12(44 \%)$ & $6(43 \%)$ & 0 & $<0.01$ \\
\hline Unilateral & $6(15 \%)$ & $3(10 \%)$ & $3(21 \%)$ & 0 & 0.54 \\
\hline Bilateral & $12(29 \%)$ & $9(33 \%)$ & $3(21 \%)$ & 0 & 0.02 \\
\hline $\begin{array}{l}\text { New diagnosis on } \\
\text { screening }\end{array}$ & $21(51 \%)$ & $11(41 \%)$ & $10(71 \%)$ & $1(7 \%)$ & 0.03 \\
\hline Unilateral & $14(34 \%)$ & $5(19 \%)$ & $9(64 \%)$ & 0 & 0.14 \\
\hline Bilateral & $7(17 \%)$ & $6(20 \%)$ & $1(7 \%)$ & $1(7 \%)$ & 0.39 \\
\hline Recurrence after release & $14(34 \%)$ & $9(33 \%)$ & $5(36 \%)$ & 0 & 0.02 \\
\hline Unilateral & $9(22 \%)$ & $6(22 \%)$ & $3(21 \%)$ & 0 & 0.07 \\
\hline Bilateral & $5(12 \%)$ & $3(11 \%)$ & $2(14 \%)$ & 0 & 0.54 \\
\hline \multicolumn{6}{|l|}{ Ulnar neuropathy } \\
\hline Any ulnar neuropathy & $14(34 \%)$ & $11(41 \%)$ & $3(21 \%)$ & $3(20 \%)$ & 0.31 \\
\hline $\begin{array}{l}\text { Onset before wtATTR } \\
\text { diagnosis }\end{array}$ & $1(2 \%)$ & $1(3 \%)$ & 0 & $1(7 \%)$ & 0.99 \\
\hline Unilateral & $1(2 \%)$ & $1(3 \%)$ & 0 & $1(7 \%)$ & 0.99 \\
\hline Bilateral & 0 & 0 & 0 & 0 & 0.99 \\
\hline $\begin{array}{l}\text { New diagnosis on } \\
\text { screening }\end{array}$ & $13(32 \%)$ & $10(37 \%)$ & $3(21 \%)$ & $2(13 \%)$ & 0.16 \\
\hline Unilateral & $8(20 \%)$ & $6(22 \%)$ & $2(14 \%)$ & $1(7 \%)$ & 0.39 \\
\hline Bilateral & $5(12 \%)$ & $4(15 \%)$ & $1(7 \%)$ & $1(7 \%)$ & 0.64 \\
\hline
\end{tabular}

CTS, carpal tunnel syndrome; wtATTR, wild-type transthyretin amyloidosis.

*Comparison between wtATTR patients without comorbidities associated with polyneuropathy and controls.

referred for carpal tunnel release surgery. A smaller subset of patients went on to have imaging investigations, muscle biopsy and surgical consultation. Over one-third of patients received neurologic follow-up, which for some included specialised multidisciplinary care. These findings suggest that peripheral neuropathy is more common than previously described among wtATTR patients, and that screening offered at the time of diagnosis results in important changes to patient management.

At our centre, routine neuropathy screening led to the early diagnosis of polyneuropathy, with $29 \%$ of all patients screened being diagnosed with mild disease. Early diagnosis of polyneuropathy in this population may facilitate early intervention with emerging TTR-targeted therapies, which have been shown to slow the progression of polyneuropathy and improve the quality of life in hereditary forms of amyloidosis, although this has not yet been demonstrated for wtATTR patients with neuropathy. ${ }^{18}$ Some
TTR-targeted therapies have been observed to have better efficacy in mild polyneuropathy compared to severe disease, further supporting the importance of early diagnosis. ${ }^{18}$ Routine neuropathy screening also facilitated the diagnosis of CTS at mild and moderate stages. Most patients in our cohort with a new diagnosis of CTS were referred for carpal tunnel release surgery. Early carpal tunnel release is favoured over delayed release as a means of preventing permanent median nerve damage. Pain, sensory abnormalities and hand function improve more quickly and completely when patients are diagnosed and treated early with surgery. ${ }^{19}$ Symptom management is integral to the treatment of ATTR patients, as it can significantly improve the quality of life. ${ }^{16}$

We observed that polyneuropathy is more common in wtATTR patients than previously reported. To date, few studies have commented on the prevalence of symptomatic polyneuropathy in this population, ${ }^{3,4,6,7}$ and none have addressed 


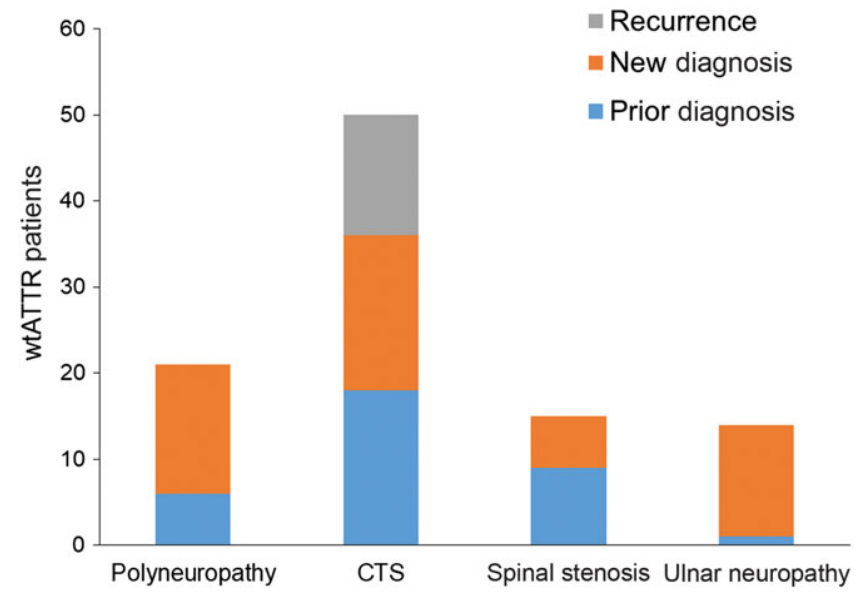

Figure 2: Frequency of prior (pre-existing) and new (diagnosed at the time of neuropathy screening) neurologic diagnoses among patients with wild-type transthyretin amyloidosis (wtATTR). Patients with a prior diagnosis of carpal tunnel syndrome (CTS) with recurrence detected at the time of neuropathy screening are also represented.

asymptomatic disease. One study of 19 wtATTR patients found that $12 \%$ had symptomatic polyneuropathy ${ }^{4}$; another reported that $6 \%$ of the 31 wtATTR patients studied had symptomatic disease. $^{3}$ In our study of 41 patients, $29 \%$ had symptomatic polyneuropathy and an additional $22 \%$ had asymptomatic disease. A more recent case series of five wtATTR patients with symptomatic polyneuropathy found that most had sensorimotor polyneuropathy, with a median PND score of I. ${ }^{6}$ Similarly, our patients had a median PND score of I; however, the majority had a pure sensory polyneuropathy. Furthermore, our study reports that wtATTR with more advanced HF defined as NYHA functional class III-IV was associated with the presence of polyneuropathy, a finding not previously reported. This further demonstrates the multisystem nature of wtATTR, suggesting more advanced disease increases the risk for neuropathy development. Spinal stenosis was also common in our cohort, with $37 \%$ having either a prior history or a new diagnosis of spinal stenosis following screening. This is compared to $13 \%$ reported in a previous study. ${ }^{3}$ Symptomatic CTS was seen in $68 \%$ of our patients; Nakagawa et al. also reported that $68 \%$ of wtATTR patients had symptomatic $\mathrm{CTS},{ }^{3}$ while $\mathrm{Ng}$ et al. reported a slightly lower prevalence (41\%). ${ }^{4}$ To our knowledge, the prevalence of ulnar neuropathy in wtATTR patients has never been reported. Symptomatic ulnar neuropathy was seen in $7 \%$ of our patients, while $27 \%$ had asymptomatic disease. The incidence of ulnar neuropathy in our patients was higher than that reported in previous studies looking at age-specific annual incidence (25/ 100,000 in individuals older than 79 and 35/100,000 in those aged $70-79$ years), ${ }^{20}$ suggesting that wtATTR may be a risk factor for this condition.

\section{Limitations}

Significant limitations of this study include the retrospective single-centre cohort design and small sample size; therefore, the presence of bias cannot be excluded. Neurologists were not blinded to clinical data. Multiple definitions of polyneuropathy have been proposed in the current literature; therefore, it is possible that the use of an alternate definition would influence the results. For patients with a prior neurologic diagnosis made before their wtATTR diagnosis, detailed neurologic data prior to their wtATTR diagnosis relating to disease progression were not available. At our centre, nerve biopsy is not routinely pursued to confirm amyloid deposition in milder and/or slowly progressive disease due to the potential risks involved. Therefore, histopathologic confirmation of amyloid neuropathy was unavailable in all but one patient included, and other causes of neuropathy cannot be excluded. Similarly, histopathological confirmation of amyloid deposition in the nerves or ligamentum flavum for patients diagnosed with spinal stenosis was not available. The prevalence of comorbidities known to cause peripheral neuropathy in some wtATTR patients included in this study confounds the ability to determine causality for the neurologic findings reported. Although even wtATTR without such comorbidities demonstrated neuropathy, our small sample size precludes controlling for these comorbidities. NCS is relatively insensitive for early neuropathic changes associated with disease, and therefore this may have been missed in such patients in our study. The sensitivity of our electrodiagnostic criteria in patients over 80 years of age is uncertain, as the normative values we used in this population had wide variability. ${ }^{14,15}$ These criteria were used to improve specificity, as the amplitudes of common SNAPs and CMAPs are known to decline significantly with age irrespective of pathology. ${ }^{12,15}$ Another limitation is that our control group was referred for clinically indicated neurologic evaluation of possible myasthenia gravis, while our wtATTR patients underwent neurologic evaluation for screening purposes. Our age-matched control group sample size was also small and therefore the incidence of neuropathic conditions may be underrepresented. Lastly, ATTR patients are at risk of autonomic dysfunction; however, formal quantitative autonomic function testing was not available.

\section{Conclusions}

Our study shows that neuropathy is a prevalent finding in wtATTR patients and indicates that routine neurologic evaluation may facilitate early detection and direct appropriate management. We observed a higher prevalence of polyneuropathy and ulnar neuropathy than previously reported, along with an anticipated high prevalence of CTS and spinal stenosis. Such assessment resulted in a change in management, including offering subsequent diagnostic work-up and/or therapeutic intervention for almost half of all patients screened. Progressive HF was associated with the presence of polyneuropathy. Based on these findings, routine screening may have value in this population, especially for patients with more advanced disease. Further prospective studies involving a larger cohort of patients with biopsy-proven amyloid neuropathy, standardised neurologic management algorithms and comparison with healthy age-matched controls are needed to verify these results.

\section{Disclosures}

Dr. Fine has received consulting fees and research support from Pfizer, Akcea and Alnylam. Dr. Hahn has received consulting fees from Pfizer, Akcea and Alnylam. The other authors have no conflicts of interest to declare.

\section{Statement of Authorship}

AR participated in study design, data collection and analysis, and manuscript preparation. SC and LK participated in study 
design and manuscript preparation. $\mathrm{CH}$ and $\mathrm{NMF}$ participated in study design, data analysis and manuscript preparation.

\section{REFERENCES}

1. Nativi-Nicolau J, Maurer MS. Amyloidosis cardiomyopathy: update in the diagnosis and treatment of the most common types. Curr Opin Cardiol. 2018;33(5):571-9.

2. Ruberg FL, Grogan M, Hanna M, Kelly JW, Maurer MS. Transthyretin amyloid cardiomyopathy: JACC state-of-the-art review. J Am Coll Cardiol. 2019;73(22):2872-91.

3. Nakagawa M, Sekijima Y, Yazaki M, et al. Carpal tunnel syndrome: a common initial symptom of systemic wild-type ATTR (ATTRwt) amyloidosis. Amyloid. 2016;23(1):58-63.

4. Ng B, Connors LH, Davidoff R, Skinner M, Falk RH. Senile systemic amyloidosis presenting with heart failure: a comparison with light chain-associated amyloidosis. Arch Intern Med. 2005;165(12):1425-9.

5. Yanagisawa A, Ueda M, Sueyoshi T, et al. Amyloid deposits derived from transthyretin in the ligamentum flavum as related to lumbar spinal canal stenosis. Mod Pathol. 2015;28(2):201-7.

6. Zivkovic S, Soman P, Lacomis D. Late-onset peripheral neuropathy in patients with wild type transthyretin amyloidosis (wtATTR). Amyloid. 2019; 27(22):1-2.

7. Sekijima Y, Uchiyama S, Tojo K, et al. High prevalence of wildtype transthyretin deposition in patients with idiopathic carpal tunnel syndrome: a common cause of carpal tunnel syndrome in the elderly. Hum Pathol. 2011;42(11):1785-91.

8. Gillmore JD, Maurer MS, Falk RH, et al. Nonbiopsy diagnosis of cardiac transthyretin amyloidosis. Circulation. 2016;133(24):2404-12.

9. Vrana JA, Gamez JD, Madden BJ, Theis JD, Bergen HR, 3rd, Dogan A. Classification of amyloidosis by laser microdissection and mass spectrometry-based proteomic analysis in clinical biopsy specimens. Blood. 2009;114(24):4957-9.
10. Werner RA, Andary M. Electrodiagnostic evaluation of carpal tunnel syndrome. Muscle Nerve. 2011;44(4):597-607.

11. American Association of Electrodiagnostic Medicine, Campbell WW. Guidelines in electrodiagnostic medicine. Practice parameter for electrodiagnostic studies in ulnar neuropathy at the elbow. Muscle Nerve Suppl. 1999;8:S171-205.

12. England JD, Gronseth GS, Franklin G, et al. Distal symmetrical polyneuropathy: definition for clinical research. Muscle Nerve. 2005;31(1):113-23.

13. Chen S, Andary M, Buschbacher R, et al. Electrodiagnostic reference values for upper and lower limb nerve conduction studies in adult populations. Muscle Nerve. 2016;54(3):371-7.

14. Falco FJ, Hennessey WJ, Goldberg G, Braddom RL. Standardized nerve conduction studies in the lower limb of the healthy elderly. Am J Phys Med Rehabil.] 1994;73(3):168-74.

15. Falco FJ, Hennessey WJ, Braddom RL, Goldberg G. Standardized nerve conduction studies in the upper limb of the healthy elderly. Am J Phys Med Rehabil. 1992;71(5):263-71.

16. Adams D. Recent advances in the treatment of familial amyloid polyneuropathy. Ther Adv Neurol Disord. 2013;6(2):129-39.

17. Yamamoto S, Wilczek HE, Nowak G, et al. Liver transplantation for familial amyloidotic polyneuropathy (FAP): a single-center experience over 16 years. Am J Transplant. 2007;7(11): 2597-604.

18. Hawkins PN, Ando Y, Dispenzeri A, Gonzalez-Duarte A, Adams D, Suhr OB. Evolving landscape in the management of transthyretin amyloidosis. Ann Med. 2015;47(8): 625-38.

19. Musad M, Rashid M, Malik SA, Khan MI, Sarwar S. Does the duration and severity of symptoms have an impact on relief of symptoms after carpal tunnel release? J Brachial Plex Peripher Nerve Inj. 2019;14:e1-8.

20. Mondelli M, Giannini F, Ballerini M, Ginanneschi F, Martorelli E. Incidence of ulnar neuropathy at the elbow in the province of Siena (Italy). J Neurol Sci. 2005;234(1-2):5-10. 